\title{
Stability properties and cross coupling performance of the control allocation scheme CAPIO
}

\author{
Yildiray Yildiz* \\ U. C. Santa Cruz, NASA Ames Research Center, Moffett Field, CA 94035, USA \\ Ilya V. Kolmanovsky ${ }^{\dagger}$ \\ University of Michigan, 1320 Beal Avenue Ann Arbor, MI 48109, USA
}

\begin{abstract}
This paper presents a stability analysis and an application of a recently developed algorithm to recover from Pilot Induced Oscillations (CAPIO). When actuators are ratesaturated due to either an aggressive pilot command, high gain of the flight control system or some anomaly in the system, the effective delay in the control loop may increase. This effective delay increase manifests itself as a phase shift between the commanded and actual system signals and can instigate Pilot Induced Oscillations (PIO). CAPIO reduces the effective time delay by minimizing the phase shift between the commanded and the actual attitude accelerations. To establish theoretical results pertinent to CAPIO stability analysis, a scalar case is presented. In addition, we present simulation results for aircraft with cross-coupling which demonstrates the potential of CAPIO serving as an effective PIO handler in adverse conditions.
\end{abstract}

\section{Introduction}

A pilot induced oscillation (PIO) can be described as "sustained or uncontrollable oscillations resulting from efforts of the pilot to control the aircraft"1,2 or "inadvertent, sustained aircraft oscillation which is the consequence of an abnormal joint enterprise between the aircraft and the pilot". ${ }^{3}$ The main commonality between various definitions is that there is an undesired, sustained oscillation due to a pilot-aircraft dynamic coupling and interaction. There are several possible instigators of PIOs such as rate saturated actuators, high gain pilot/controller, system delays and phase lags. The authors previously proposed a Control Allocation technique to recover from Pilot Induced Oscillations (CAPIO $)^{4}$ due to actuator rate saturation. In this paper, a comparison between conventional control allocation and CAPIO is given in the case of a PIO event when the aircraft has inertial cross coupling. Moreover, a stability analysis is provided for systems with CAPIO.

Actuator rate saturation is a phenomenon that is frequently observed during PIO events and has led to several crashes. A comprehensive overview of the effect of rate limiting on PIOs is given by Klyde and Mitchell. ${ }^{5}$ Figure 1 presents a basic model for a rate limited actuator, ${ }^{5}$ where $u$ is the input to the actuator and $\delta$ is the actual actuator deflection. Without the rate limit, this dynamics is simply a first order lag, which is often used as an approximate model for actuators. Figure $2^{5-7}$ shows time evolutions of input-output signals of such a rate saturated actuator, where $u_{c}=u$ represents the pilot command. Gain reduction and an increase in effective time delay are two detrimental results of rate saturation, as seen from this figure.

There are several successful approaches in the literature that address eliminating the effective time delay. Differentiate-limit-integrate (DLI) approach $^{8-12}$ is one of the approaches that is implemented using a "software rate limiter" as shown in Fig. 3, where the software limiter is placed between the command signal and the input signal to the actuator. This method eliminates the effective time delay introduced by the rate saturation as seen in Fig. 4 and hence the onset of a PIO can be avoided. The DLI method proved very successful both in simulation and actual flight tests. It has, however, some deficiencies such as introduction of a bias and susceptibility to noise. See Fig. 5. These problems may be handled using different techniques such as filtering and resetting/retrimming. ${ }^{9-11}$ See also Ref. 12 for more improved results.

\footnotetext{
*Associate scientist, University Affiliated Research Center, AIAA Member.

${ }^{\dagger}$ Professor, Department of Aerospace Engineering, AIAA Member.
} 


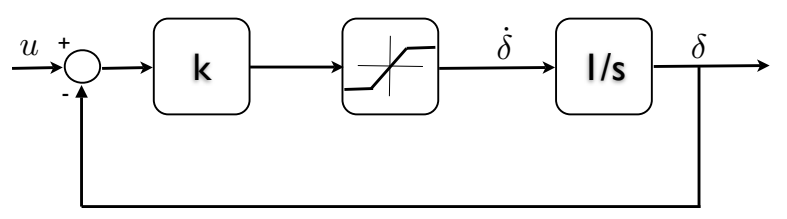

Figure 1. Actuator model with rate saturation.

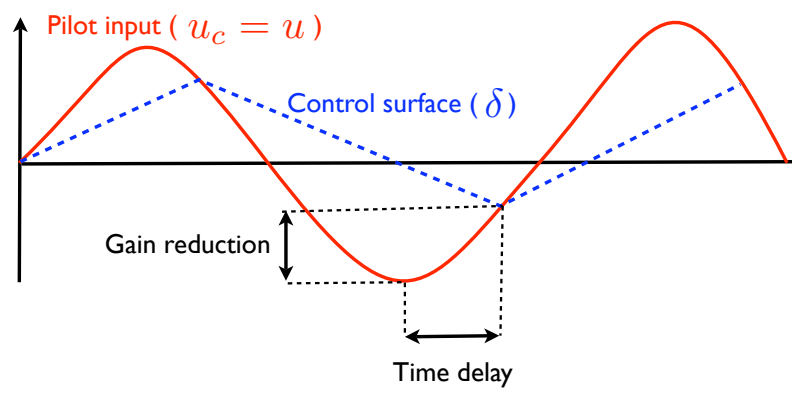

Figure 2. Input $u$ and output $\delta$ of a rate saturated actuator.

There are also other methods for dealing with the effective time delay based on manipulating the input signal: Smith and Edwards ${ }^{13}$ used a nonlinear adaptive filter to attenuate the pitch stick shaping function gain depending on the magnitude and frequency of the pilot input and the rate limits of the elevators. This approach was successfully implemented in Space Shuttle Enterprise ALT-5 and no further pitch PIO events were reported in the open literature since the 1977 event. ${ }^{5}$ Koper ${ }^{14}$ developed a phase compensating filter which reverses the direction of a rate saturated actuator when the derivatives of the input and the output signals have opposite sign and when the absolute value of the error is increasing. Rundqwist and Hillgren ${ }^{15}$ developed a similar actuator output reversing logic wherein a feedback signal is passed through a lowpass filter. This approach also proved to be successful in actual flight tests. Hanke ${ }^{16}$ developed another phase compensator for a rate limiting element without using any feedback or logic but employing describing function ${ }^{17}$ relationships. This filter also performed successfully in flight tests.

To the best of authors' knowledge, all the previously reported successful implementation results were for SISO applications without any redundant actuators. Consider the closed loop flight control structure in Fig. 6, where the pilot is also in the loop. In this configuration, the pilot task may be to track an altitude reference $r$, by getting altitude measurement feedback $y$, and making necessary corrections via a pilot stick which gives pitch rate commands $u_{c}$ as a reference to the inner flight controller. The inner controller may also be responding to roll and yaw rate commands at the same time. So, the "pilot command" $u_{c}$ can be a vector of three elements. The controller then calculates the necessary attitude accelerations $v \in \Re^{3}$ and then control allocator allocates the available actuators $u \in \Re^{m}, m>3$, to achieve these desired accelerations while possibly satisfying secondary objectives like drag minimization. In this scenario, it is not obvious where and how to use the DLI software limiter. An extension of using the DLI method to multi-input multi-output (MIMO) applications is given by Hess and Snell, ${ }^{9}$ however, the authors had to use "ganged" actuators for successful implementation. Ganging of the actuators, however, prevents the use of redundant actuators for secondary objectives like drag minimization or reconfiguration after a failure. In addition, ganging becomes

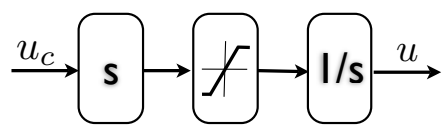

Figure 3. Software rate limiter. 


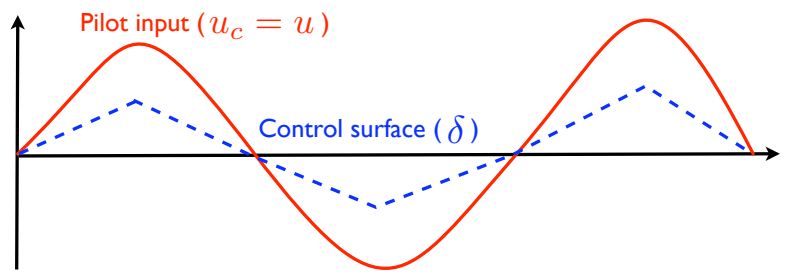

Figure 4. Input $u_{c}$ and output $\delta$ of a rate saturated actuator with a preceding software rate limiter.

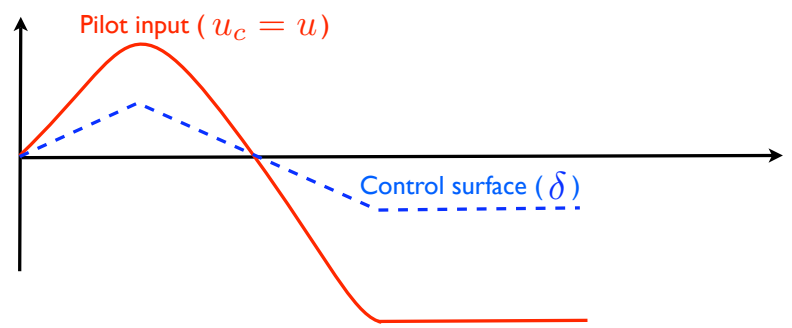

Figure 5. Bias caused by the differentiate-limit-integrate approach

more cumbersome as the number of actuators increases. ${ }^{18}$

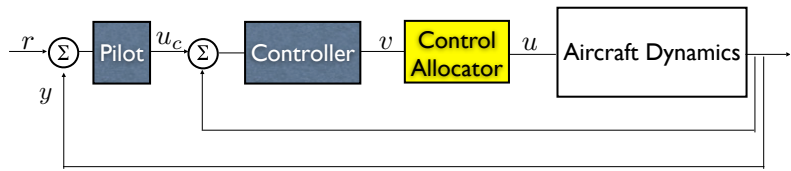

Figure 6. Overall SISO system structure

It is noted that Durham and Bordignon ${ }^{19}$ extended the direct control allocation scheme to make it easier to implement for the case of rate-limited actuators and consequently ended up with a "momentrate allocation" scheme. Although there is no implementation result showing a PIO preventing example in this study, this control allocation scheme has a potential to handle PIOs despite being more complicated than CAPIO. Furthermore, the technique ${ }^{19}$ needs the calculation of a moment rate set which can introduce additional computational intensity.

The control allocation method CAPIO, previously proposed by the authors, ${ }^{4}$ is suitable for MIMO applications in the presence of redundant actuators. The main idea behind CAPIO is to minimize the phase lag introduced into the system due to rate saturation by minimizing the error between the derivatives of desired and actual total control effort vectors as well as minimizing the error between them, using constrained optimization techniques. To achieve this goal, for example in a SISO case, one needs to minimize the phase lag between the pilot input and the control surface deflection. On the other hand, in a MIMO case, where there are multiple inputs and outputs, one needs to pinpoint where exactly the phase lag is being introduced into the system. For example, in a scenario where the flight control system produces the desired rate accelerations and a control allocator distributes these commands to redundant actuators using some predefined optimization routine, it makes more sense to minimize the phase lag between the desired and achieved accelerations than concentrating on individual actuator signals. It is noted that merely having a control allocation scheme that takes into account the rate limits of the actuators as constraints can not prevent phase shift between the desired and achieved accelerations when saturation is unavoidable, and thus may not be able to handle a PIO situation. It was shown in Ref. 4, where PIOs were not prevented with conventional control allocation techniques, that the onset of these PIOs could be prevented using CAPIO.

In this paper, we first present new results for the case in which an aircraft is assumed to have inertial cross-coupling. Cross coupling between the lateral and longitudinal equations of aircraft becomes dominant in cases where the weight is concentrated along the fuselage as the aircraft's wings became thinner and 
shorter, causing a shift of weight. ${ }^{20}$ A similar effect can also be observed in damaged aircraft where the weight shift may occur, for example, as a result of a more than $25 \%$ wing loss. ${ }^{21}$ In the event of cross coupling, a PIO becomes more dangerous since an onset of a PIO in one axis can effect other axes resulting in a catastrophic failure. To the best of authors' knowledge, no PIO prevention technique were tested for these cases. It is shown in this paper that CAPIO has the potential to help the aircraft recover from PIOs in the presence of cross-coupling. Moreover, we show that a scalar closed loop system that is stable neglecting the rate limiting elements remains stable in the presence of rate limiting elements if CAPIO is used as the control allocator. The results can be extended for MIMO systems.

The organization of this paper is as follows. In Section II, flight control of an aircraft with inertial cross coupling is presented. A PIO event is created using a high gain pilot model and conventional control allocators and CAPIO are compared in terms of handling the PIO situation. In Section III, the stability analysis is given. Finally, in Section IV, a summary is given.

\section{Flight Control of an Aircraft with Inertial Cross-coupling}

To show the advantages of CAPIO, a flight control example using a simplified ${ }^{22}$ ADMIRE model ${ }^{23,24}$ is used with some modifications to simulate inertial cross coupling. This model includes redundant actuators which make the DLI method hard to apply if one does not want to gang the actuators.

The linearized aircraft model at Mach 0.22 , altitude $3000 \mathrm{~m}$ is given by

$$
\begin{aligned}
& x=\left[\begin{array}{lllll}
\alpha & \beta & p & q & r
\end{array}\right]^{T}-x_{\text {lin }}, \\
& y=C x=\left[\begin{array}{lll}
p & q & r
\end{array}\right]^{T}-y_{\text {lin }}, \\
& \delta=\left[\begin{array}{llll}
\delta_{c} & \delta_{r e} & \delta_{l e} & \delta_{r}
\end{array}\right]^{T}-\delta_{\mathrm{lin}}, \\
& u=\left[\begin{array}{llll}
u_{c} & u_{\mathrm{re}} & u_{l e} & u_{r}
\end{array}\right]^{T}-u_{\mathrm{lin}} \\
& {\left[\begin{array}{c}
\dot{x} \\
\dot{\delta}
\end{array}\right]=\left[\begin{array}{cc}
A & B_{x} \\
0 & -B_{\delta}
\end{array}\right]\left[\begin{array}{l}
x \\
\delta
\end{array}\right]+\left[\begin{array}{c}
0 \\
B_{\delta}
\end{array}\right] u,}
\end{aligned}
$$

where $\alpha, \beta, p, q$ and $r$ are the angle of attack, sideslip angle, roll rate, pitch rate and yaw rate, respectively. $\delta$ and $u$ represent the actual and the commanded control surface deflections, respectively. Control surfaces are canard wings, right and left elevons and the rudder. (.) lin refers to values at the operating points where the linearization was performed. The actuators have the following position and rate limits

$$
\begin{aligned}
& \delta_{c} \in[-55,25] \times \frac{\pi}{180} ; \quad \delta_{r e}, \delta_{l e}, \delta_{r} \in[-30,30] \times \frac{\pi}{180} \\
& \dot{\delta}_{c}, \dot{\delta}_{r e}, \dot{\delta}_{l e}, \dot{\delta}_{r} \in[-70,70] \times \frac{\pi}{180}
\end{aligned}
$$

and have first order dynamics with a time constant of 0.05 seconds. It is noted that the position limits given are the same as the ones given by Harkegard ${ }^{22}$ but the rate limits are assumed to illustrate CAPIO properties.

To make this model suitable for control allocation implementation, the actuator dynamics are neglected and the control surfaces are viewed as pure moment generators and their influence on $\dot{\alpha}$ and $\dot{\beta}$ is neglected. It is noted that the actuators dynamics are present during the simulations, i.e. they are neglected only during the control allocation algorithm derivation. These assumptions lead to the following approximate model

$$
\begin{aligned}
\dot{x} & =A x+B_{u} u=A x+B_{v} v, \\
v & =B u,
\end{aligned}
$$

where

$$
\begin{aligned}
& B_{u}=B_{v} B, \quad B_{v}=\left[\begin{array}{c}
0_{2 \times 3} \\
I_{3 \times 3}
\end{array}\right], \\
& A=\left[\begin{array}{ccccc}
-0.5432 & 0.0137 & 0 & 0.9778 & 0 \\
0 & -0.1179 & 0.2215 & 0 & -0.9661 \\
0 & -10.5128 & -0.9967 & 0 & 0.6176 \\
2.6221 & -0.0030 & 0 & -0.5057 & 0 \\
0 & 0.7075 & -0.0939 & 0 & -0.2127
\end{array}\right],
\end{aligned}
$$




$$
B=\left[\begin{array}{cccc}
0 & -4.2423 & 4.2423 & 1.4871 \\
1.6532 & -1.2735 & -1.2735 & 0.0024 \\
0 & -0.2805 & 0.2805 & -0.8823
\end{array}\right]
$$

The virtual (total) control effort, $v$, consists of the angular accelerations in roll, pitch and yaw. To simulate the effects of inertial cross-coupling, we modify the $A$ matrix so that a change in pitch angular velocity creates a moment in roll and yaw axes:

$$
A=\left[\begin{array}{ccccc}
-0.5432 & 0.0137 & 0 & 0.9778 & 0 \\
0 & -0.1179 & 0.2215 & 0 & -0.9661 \\
0 & -10.5128 & -0.9967 & 1 & 0.6176 \\
2.6221 & -0.0030 & 0 & -0.5057 & 0 \\
0 & 0.7075 & -0.0939 & 0.1 & -0.2127
\end{array}\right]
$$

In this flight control example the pilot task is to track a given pitch angle reference, $\theta_{d}$, using a pitch rate, $q_{d}$, stick. In addition, roll rate, $p$, and the yaw rate, $r$, are to be controlled independently to track their references $p_{d}$ and $r_{d}$. The overall system configuration is given in Fig. 7 .

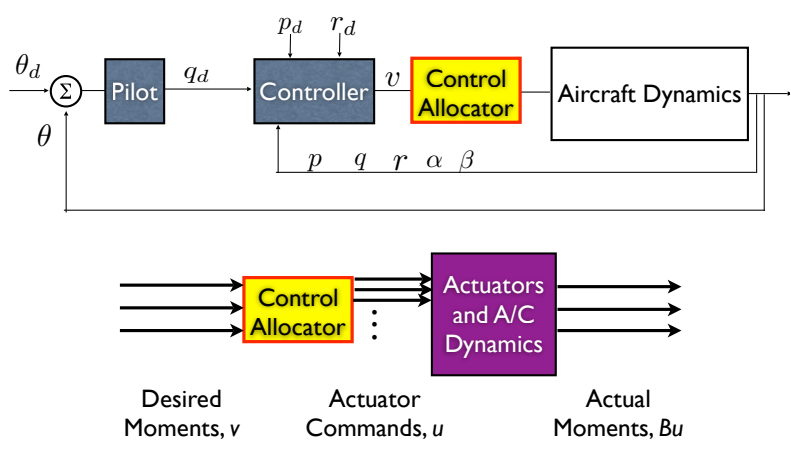

Figure 7. Overall MIMO system structure

The inner controller is a dynamic inversion controller which uses $q_{d}, p_{d}$ and $r_{d}$ as references and produces the necessary attitude accelerations, $v \in \Re^{3}$, to track these references. Dynamic inversion control laws, $v$, make the closed loop dynamics follow a desired reference model

$$
\dot{y}_{m}=A_{m} y_{m}+B_{m} r_{m}
$$

where $y_{m}=\left[\begin{array}{lll}p_{m} & q_{m} & r_{m}\end{array}\right]^{T}$ represents the desired output vector and $r_{m}=\left[\begin{array}{lll}p_{d} & q_{d} & r_{d}\end{array}\right]^{T}$ is the reference input vector. In this example, $A_{m}=-2 \times I_{3 \times 3}$ and $B_{m}=2 \times I_{3 \times 3}$. Reference model tracking can be achieved by inverting the dynamics ${ }^{18}$ as

$$
v=\left(C B_{v}\right)^{-1}\left[A_{m} y+B_{m} r_{m}-C A x\right] .
$$

The control allocator distributes this total control effort, $v$, to individual control surfaces via the actuator commands, $u \in \Re^{4}$. The control surfaces then produce actual attitude accelerations, $B u$, where $B$ is the control input matrix. The pilot is modeled as a pure gain for simplicity.

\section{II.A. Flight control with conventional control allocation}

The conventional control allocation used in this example minimizes the following objective function

$$
J=\|B u-v\|_{2}^{2}+\epsilon\|u\|_{2}^{2}
$$

subject to $\max \left(\dot{u}_{\min } T+u_{k-1}, u_{\min }\right) \leq u \leq \min \left(\dot{u}_{\max } T+u_{k-1}, u_{\max }\right)$, where $T$ is the sampling interval. It is noted that norms, instead of square-norms, can be used in the objective function. Note that (7) is in the form of a typical objective function used in conventional control allocators, ${ }^{18}$ where the main objective 
is to minimize the error between the desired and the actual total control efforts. As $\epsilon \rightarrow 0$, minimizing (7) becomes equivalent to achieving the main objective explained above and picking the solution that gives the minimum control surface deflection, among different solutions. In this example $\epsilon=10^{-5}$.

Figure 8 presents the simulation result with the conventional control allocation where the pilot receives a step pitch angle reference at $t=3$ seconds and the inner controller receives a pulse yaw rate reference at $t=0.5$ seconds and a zero roll rate reference at all times. The pilot is aggressive and has a gain of 4.11. Because of this high gain, the aircraft goes into a divergent PIO in the pitch axis. In addition, inertial cross coupling causes dangerous oscillations in the roll axis, which finally diverges. Yaw axis also becomes unstable. Canard wings and the ailerons saturate both in position and the rate. The results of saturation can best be observed as a phase shift between the desired pitch acceleration $v_{2}$ and the actual pitch acceleration created by the control surfaces $B u_{2}$. This phase shift, or the effective time delay, is something that is almost always observed in PIO events due to actuator saturation.
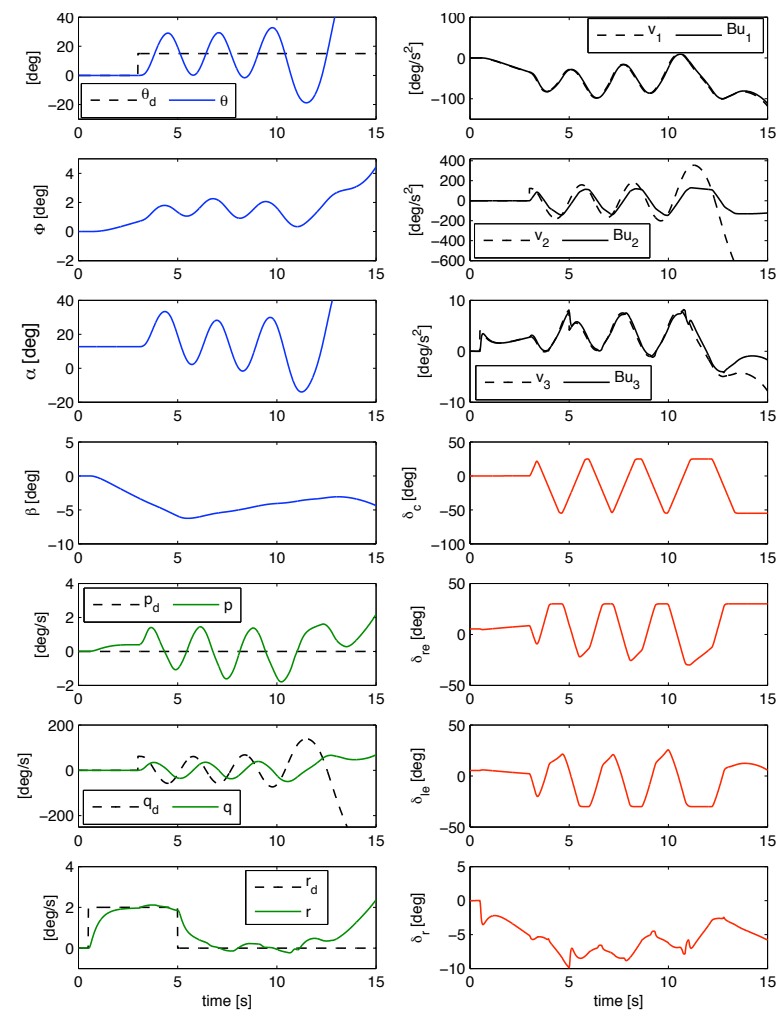

Figure 8. Pitch and roll angles $\theta$ and $\phi$, aircraft states $x$, on the left. Desired (commanded) and actual attitude accelerations $v$ and $B u$, and the control surface deflections $\delta$, on the right, when a conventional control allocator is used.

\section{II.B. Flight control with CAPIO}

To recover from a PIO event, CAPIO forces the virtual (total) control effort $v$, to be in phase with the actual control effort $B u$ produced by the actuators. To achieve this, a derivative error term is added to objective function (7) to obtain the following CAPIO objective function

$$
J^{\prime}=\|B u-v\|_{2}^{2}+\left\|W_{d}(B \dot{u}-\dot{v})\right\|_{2}^{2}+\epsilon\|u\|_{2}^{2}
$$

where $W_{d} \in R^{3 \times 3}$ represents a weighting matrix on the derivative term. The cost function $J^{\prime}$ is minimized with respect to $u$, with $\dot{u}=\left(u-u^{-}\right) / T$, where $u^{-}$denotes the value of $u$ at the previous sampling instant. It is noted that with this modified objective function, the control allocator is trying to realize $\dot{v}$ as well as $v$. Very high values of $W_{d}$ make the signals, $v$ and $B u$, have approximately the same derivative at all times, which eliminates the phase lag completely but causes a bias as shown in Fig. 5. On the other hand, very small values of $W_{d}$ may not be sufficient for the control allocator to be any different than the conventional one and thus does not prevent PIOs. Therefore, the designer needs to decide on suitable values of $W_{d}$ that 
minimize the phase lag and at the same time prevent a bias. As an alternative, the designer can choose to "activate" $W_{d}$, i.e. set it to a constant matrix, only when it is needed, and keep it a 0 matrix at all other times. The latter approach is taken in this paper, assuming that a PIO detection algorithm is available on board of the aircraft. $W_{d}$ can also be utilized for axis prioritization.

The objective function (8) needs to be transformed into a form that can be minimized numerically. To achieve this goal, the derivatives in the objective function are approximated as $\dot{u}=\left(u-u^{-}\right) / T$. After some algebra, (8) can be rewritten as

$$
\begin{aligned}
J^{\prime}= & u^{T}\left(B^{T} T^{2} B+B^{T} R B+\epsilon I_{4 \times 4}\right) u \\
& +2\left(-v^{T} T^{2} B-u^{-T} B^{T} R B-\dot{v}^{T} T R B\right) u \\
& +v^{T} T^{2} v+u^{-T} B^{T} R B u^{-}+2 u^{-T} B^{T} R T \dot{v} \\
& +\dot{v}^{T} T^{2} R \dot{v}
\end{aligned}
$$

subject to $\max \left(\dot{u}_{\min } T+u^{-}, u_{\min }\right) \leq u \leq \min \left(\dot{u}_{\max } T+u^{-}, u_{\max }\right)$, where $R=W_{d}^{T} W_{d}$.

Figure 9 presents the simulation result when CAPIO is used as the control allocator. All the settings including the pilot gain are the same as in the previous example with the conventional control allocation. Since CAPIO prevents the effective time delay introduction, the aircraft now recovers from the PIO and no dangerous oscillation or divergence is observed in any axis.
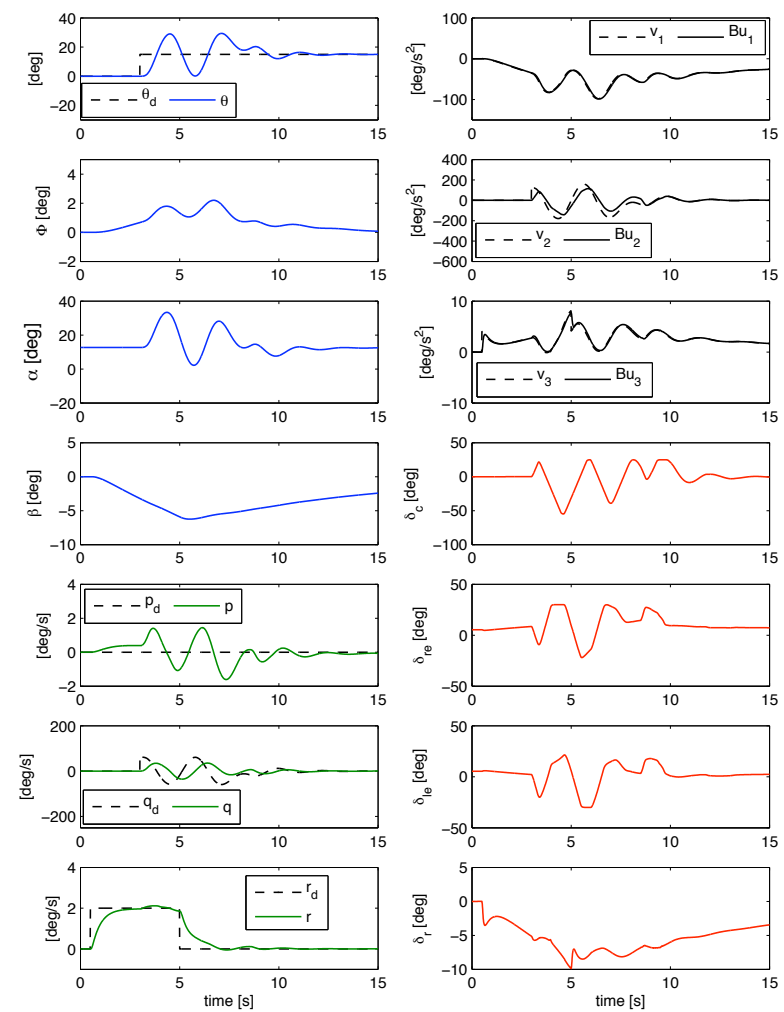

Figure 9. Pitch angle $\theta$ and aircraft states $x$, on the left. Desired (commanded) and actual attitude accelerations $v$ and $B u$, and the control surface deflections $\delta$, on the right, when CAPIO is used.

To show the difference that CAPIO makes in control effort realization, the pitch axis accelerations are presented again in Fig. 10 for both cases. It is noted as as soon as the PIO detection signal is obtained, CAPIO forces the control surfaces to produce accelerations "in phase" with the commanded accelerations, eliminating the time delay due to phase shift. When the aircraft recovers from PIO, control surfaces arrange themselves to track the commanded acceleration. The result is recovery from the PIO without any bias formation.

The constrained optimization of the cost (9) is a low dimensional quadratic programming problem with linear inequality constraints. This problem depends on a vector of parameters, specifically, on $p=$ 

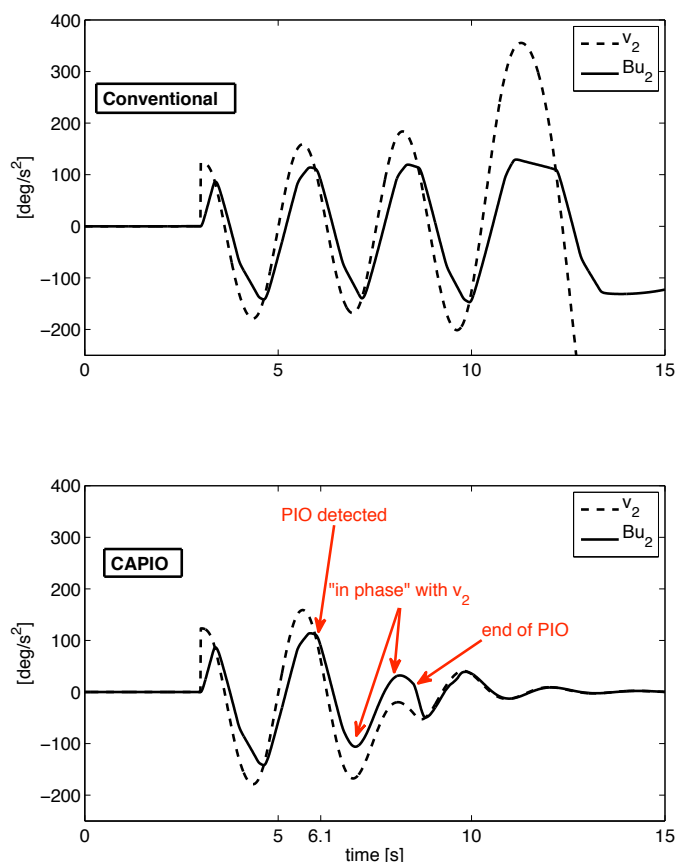

Figure 10. commanded and achieved pitch accelerations, $B u_{2}$ and $v_{2}$, in the case of a conventional control allocator (top) and CAPIO (bottom).

$\left[\begin{array}{lll}u^{-} & v & \dot{v}\end{array}\right]$. Note that the parameters enter linear in the cost and in the constraints and, hence, such a quadratic programming problem can be solved explicitly using off-line multi-parametric QP solvers. The solution is known to be a piecewise affine continuous function of the parameter vector and have the following form, $u=f_{i} p+g_{i}$, if $F_{i}+G_{i} \leq 0, i=1, \ldots, N_{r}$ where $N_{r}$ is the finite number of polyhedral regions and each region is associated with its set of linear inequality constraints and its affine map. Such an explicit solution is computed off-line and can be deployed on-line in the aircraft software using a set of simple if-then-else rules, additions, multiplications and comparisons. The need to embed a quadratic programming solver to perform constrained optimization of the cost (9) within aircraft software can thus be avoided altogether. A cross-section of the explicit solution polyhedral regions is illustrated in Fig. 11; the explicit solution has $N_{r}=223$ regions.

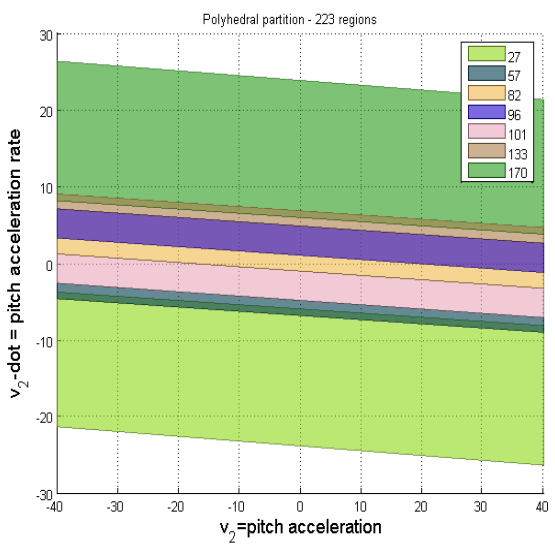

Figure 11. Cross-section of regions of explicit solution by pitch acceleration - pitch acceleration rate plane. 


\section{Stability analysis}

To simplify the stability analysis, we assume a closed loop system where the virtual control input $v$ and real control input $u$ are scalars. Furthermore, we assume that the reference input is zero. The resulting system structure is presented in Fig. 12. Consider a plant dynamics represented in state space form and a stabilizing state feedback controller:

$$
\begin{aligned}
\dot{x} & =A x+B u \\
v & =-K x,
\end{aligned}
$$

where $x \in \Re^{n}, A \in \Re^{n \times n}, B \in \Re^{n}, v$ is a scalar and $K$ is such that $(A-B K)$ has only negative eigenvalues. Note that plant is not assumed to be open loop stable.

We assume that $W_{d}$ is large enough so that CAPIO forces the commanded control input behave in the following manner:

$$
\begin{array}{ll}
\dot{u}=\operatorname{sign}(\dot{v}) l & \text { if } \quad|\dot{v}|>l \\
\dot{u}=\dot{v} & \text { otherwise }
\end{array}
$$

where $l$ is the actuator rate limit. It is noted that the second equation in (11) is due to CAPIO forcing the two signals to have the same derivative, i.e. $\dot{u}=\dot{v}$. According to the first equation in (11), $u$ is trying to follow $v$ as much as possible when actuators are saturated. As soon as the saturation ends, $u$ follows $v$ in a manner that their derivatives become equal. This behavior prevents wind up and the introduction of an effective time delay. When $W_{d}$ is switched off after the recovery, CAPIO forces $u$ to follow the commanded control input $v$, hence prevents bias formation.

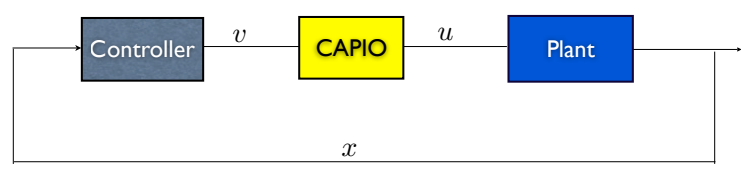

Figure 12. Closed loop system with CAPIO

It is noted that the system (10)-(11) is a SISO linear system with states $(x, u)$ and saturation at the input $v$. Its stability may be analyzed using various techniques including Lyapunov functions, describing functions, or circle/Popov criterion.

For stability analysis we assume that there is a PIO event at $t=t_{0}$ and thus the system is experiencing a sustained oscillation. The commanded control input $v$ is also in a sustained oscillation mode and causing the actuators to rate-saturate. In other words, $\dot{v}$ is oscillating past $\pm l$. Figure 13 illustrates the oscillation of $\dot{v}$ in a region containing the rate saturation limits $\pm l$.

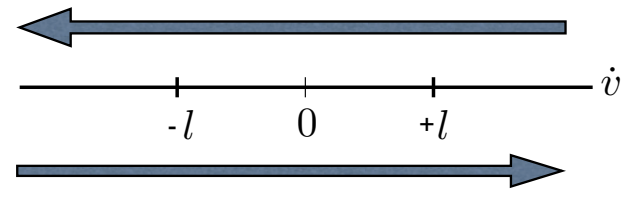

Figure 13. Oscillation of $\dot{v}$ past $\pm l$.

There is no difference between the conventional control allocators and CAPIO when $|\dot{v}|>l$. Both forces the actual control input $u$ follow the commanded control input $v$ as much as possible. However, once $|\dot{v}|<l$, CAPIO stabilizes the system for $t \geq t_{c}$. To show this, consider the closed loop system behavior in the unsaturated region by employing the second equation in (11) in the plant dynamics (10). It is noted that $u=v+d$ for a constant $d=u\left(t_{c}\right)-v\left(t_{c}\right)$ and

$$
\dot{x}=A x+B(v+d)
$$




$$
\begin{aligned}
& =A x+B(-K x+d) \\
& =(A-B K) x+B d .
\end{aligned}
$$

Defining $M \equiv A-B K$, the solution to (12) can be given as

$$
x(t)=e^{M\left(t-t_{0}\right)}\left(x\left(t_{0}\right)+M^{-1} B d\right)-M^{-1} B d
$$

Differentiating (13), we obtain

$$
\dot{x}(t)=e^{M\left(t-t_{0}\right)}\left(M x\left(t_{0}\right)+B d\right) .
$$

Therefore, if $|\dot{v}(t)| \leq l$ for $t \geq t_{c}$ for some $t_{c}$, we have

$$
\begin{aligned}
\lim _{t \rightarrow \infty} x(t) & =-M^{-1} B d, \\
\lim _{t \rightarrow \infty} \dot{x}(t) & =0, \\
\lim _{t \rightarrow \infty} \dot{v}(t) & =\lim _{t \rightarrow \infty}-K \dot{x}(t)=0 .
\end{aligned}
$$

Hence, $x(t)$ tends to $-M^{-1} B d$, whenever $|\dot{v}| \leq l$. It is noted that $-M^{-1} B d$ corresponds to a steady state error or a bias, which appears until the derivative term is switched off. Once the system recovers from PIO and the derivative term is switched off, CAPIO forces $u$ to follow $v$ and the steady state error is eliminated. This behavior can be observed in Figs. 9 and 10.

\section{III.A. Stability analysis using circle criterion}

To eliminate the assumption that the system is already in a PIO, we next use the circle criterion ${ }^{25}$ to investigate the stability of the overall closed loop system with CAPIO as the control allocator. Taking the derivative of (10) and defining $\tilde{x}=\dot{x}, \tilde{u}=\dot{u}$ and $\tilde{v}=\dot{v}$, we obtain

$$
\begin{aligned}
\dot{\tilde{x}} & =A \tilde{x}+B \tilde{u} \\
\tilde{v} & =-K \tilde{x} .
\end{aligned}
$$

Using the same definitions, we can rewrite (11) as

$$
\begin{array}{lll}
\tilde{u}=\operatorname{sign}(\tilde{v}) l & \text { if } & |\tilde{v}|>l \\
\tilde{u}=\tilde{v} & \text { otherwise. }
\end{array}
$$

Defining $\tilde{u}^{\prime}=-\tilde{u}$ and $\tilde{v}^{\prime}=-\tilde{v}$, we can represent (16) and (17) as in Fig. 14 .

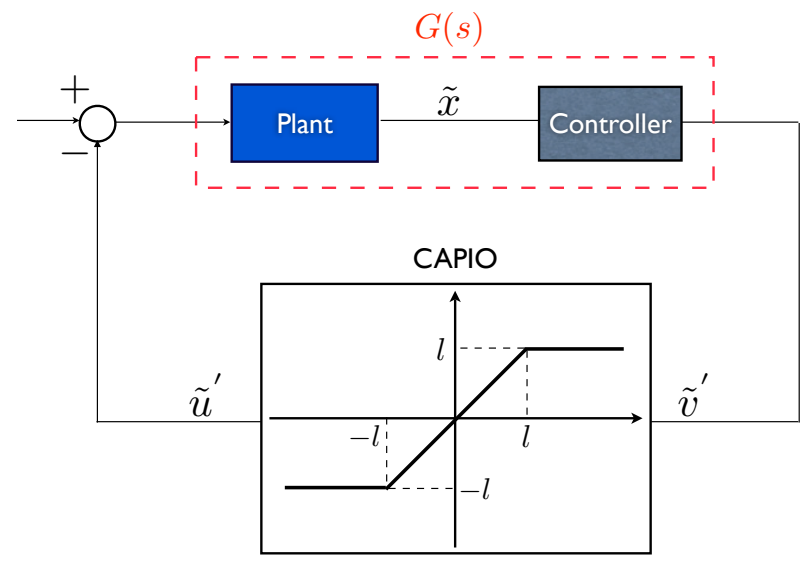

Figure 14. Closed loop system with CAPIO, reconfigured.

To investigate the stability properties of the closed loop system given in Fig. 14, we need to know the loop transfer function $G(s)$. We will use the pitch angle control system example that was given in our earlier 
paper $^{4}$ where we introduced the CAPIO without investigating the closed loop stability. We assume a short period dynamics for the plant model which is given as

$$
P(s)=\frac{1.39(s+0.306)}{s^{3}+0.805 s^{2}+1.325 s} .
$$

We also assume that the pilot (controller) is a pure gain which has a value of 1.65 . Therefore, the loop transfer function is obtained as

$$
G(s)=\frac{2.293(s+0.306)}{s^{3}+0.805 s^{2}+1.325 s} .
$$

CAPIO nonlinearity belongs to the sector $[0,1]$ (see reference 25 for the circle criterion and sector definition) but $G(s)$ is not Hurwitz. Hence, we can not obtain an absolute stability result using the circle criterion. Rather, we will give an absolute stability result with a finite domain. Figure 15 shows that CAPIO is in the sector $\left[\begin{array}{ll}\alpha & 1\end{array}\right]$ for $\tilde{v}^{\prime} \in\left[\begin{array}{ll}-l / \alpha & l / \alpha\end{array}\right]$. According to circle criterion, the closed loop system is absolutely stable if the transfer function

$$
Z(s)=\frac{1+G(w j)}{1+\alpha G(w j)}
$$

is strictly positive real (SPR). $Z(s)$ is SPR if it is Hurwitz and

$$
\operatorname{Re}\left[\frac{1+G(w j)}{1+\alpha G(w j)}\right]>0, \quad \forall w \in(-\infty, \infty) .
$$

These two requirements are satisfied if the Nyquist plot of $G(s)$ a) does not enters the disk $D(\alpha, 1)$, which is defined as the closed disk in the complex plane whose diameter is the line segment connecting the points $-(1 / \alpha)+0 j$ and $-1+0 j$, and b) encircles it $m$ times in the counterclockwise direction, where $m$ is the number of unstable poles of $G(s) .{ }^{25} G(s)$ given in (19) has no unstable roots, therefore $m=0$. Also, it can be found analytically that (21) is satisfied for $\alpha \geq 0.135$. Indeed, Fig. 16 shows that the Nyquist plot of $G(s)$ is not touching the circle $D(0.135,1)$.

To summarize, the circle criterion shows that as long as the commended control signal rate $\dot{v}$ does not exceed $l / 0.135=7.4 l$, the origin of the closed loop system, $\tilde{x}=\tilde{v}=0$, is absolutely stable, i.e. globally, uniformly asymptotically stable.

\section{III.B. Stability Analysis using Popov Criterion}

In the previous section, we found that, according to the circle criterion, the closed loop system described by (16) and (17) is absolutely stable if the rate of the control input is smaller than or equal to 7.4 times the rate limit. Although this result gives a reasonable range for the allowable control input rate, we want to obtain a more relaxed condition for absolute stability. To achieve this, we use the "Popov Criterion" 25 for stability analysis which can be less conservative compared to circle criterion in some cases.

Consider Fig. 14 with CAPIO nonlinearity. According to Popov criterion, to show that the closed loop system is absolutely stable, the nonlinearity must belong to the sector $[0, k]$ ( $\operatorname{see}^{25}$ chapter 7 for sector definition) and the following transfer function must be strictly positive real (SPR).

$$
Z Z(s)=\frac{1}{k}+(1+s \gamma) G(s)
$$

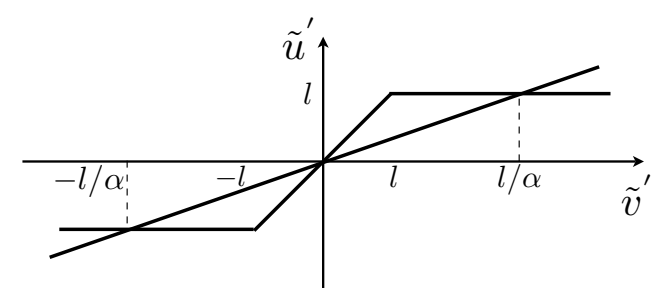

Figure 15. Sector for CAPIO nonlinearity 


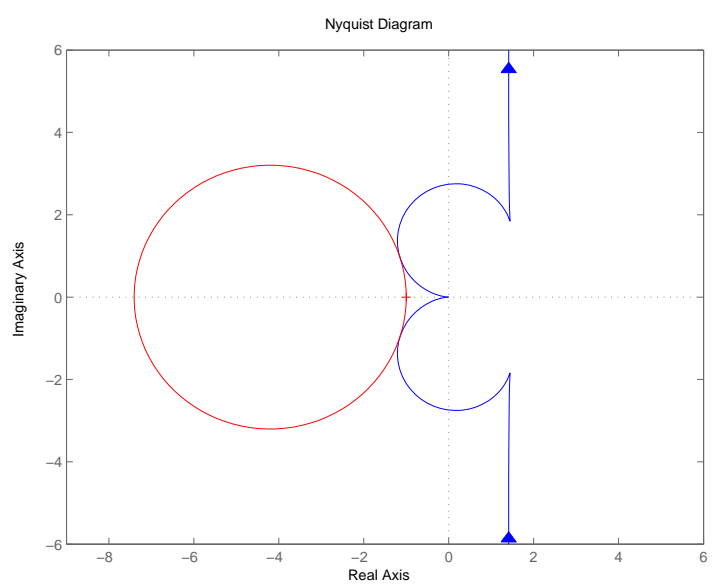

Figure 16. Circle criteria satisfied by $G(s)$

where $\gamma>0$ is such that $\left(1+\lambda_{i} \gamma\right) \neq 0$, for any eigenvalue $\lambda_{i}$ of the plant $G(s)$. For $Z Z(s)$ to be SPR, $G(s)$ must be Hurwitz. However, we know that $G(s)$, given in (19), is not Hurwitz. In addition, we are trying to find the largest sector that the nonlinearity can be accommodated, so we assume that CAPIO $\in[\alpha, \beta]$ and our goal is to find the largest $\beta$ and smallest $\alpha$ that is allowed. To represent the problem in accordance with the Popov Criterion requirements, we apply the loop transformation as shown in Fig. 17. After this transformation, we have our nonlinearity transformed to $\mathrm{CAPIO}_{\mathrm{t}} \in[0, k]$, where $k=\beta-\alpha$. In addition, our transformed linear system becomes

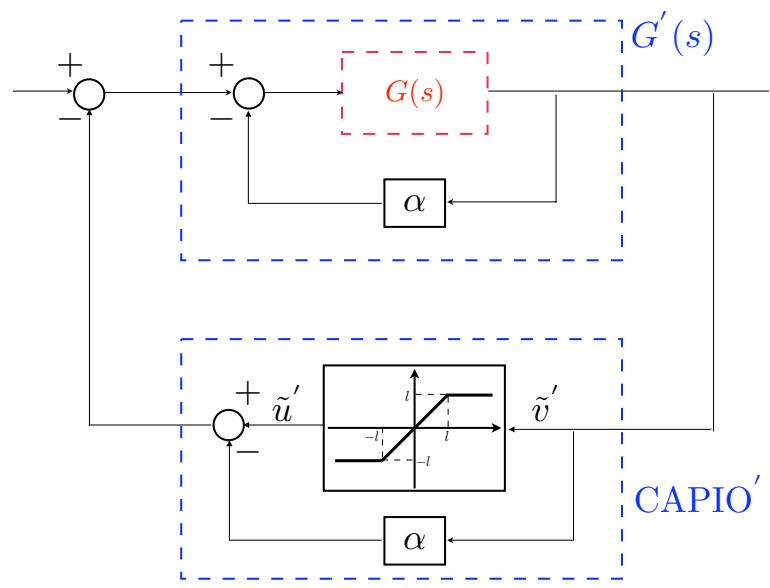

Figure 17. CAPIO $\in[\alpha, \beta]$ transformed to CAPIO $_{\mathrm{t}} \in[0, \beta-\alpha]$ via loop transformation.

$$
G_{\mathrm{t}}(s)=\frac{2.293(s+0.306)}{s^{3}+0.805 s^{2}+(1.325+2.293 \alpha) s+0.7017 \alpha} .
$$

To show that $Z Z_{\mathrm{t}}(s)=(1 / k)+(1+s \gamma) G_{\mathrm{t}}(s)$ is SPR, we need to show that

$$
\frac{1}{k}+\operatorname{Re}\left[G_{\mathrm{t}}(j \omega)\right]-\gamma \omega \operatorname{Im}\left[G_{\mathrm{t}}(j \omega)\right]>0, \quad \forall \omega \in[-\infty, \infty] .
$$

Substituting (23) into (24) we obtain, after some algebra, that

$$
\frac{1}{k}+\frac{(1.8459 \gamma-2.293) \omega^{4}+(2.4733+5.2578 \alpha+0.9298 \gamma) \omega^{2}+0.4924 \alpha}{\left(0.7017 \alpha-0.805 \omega^{2}\right)^{2}+\left(1.325 \omega+2.293 \alpha \omega-\omega^{3}\right)^{2}}>0 .
$$


It is clear that for any positive values of $k, \alpha$ and for $\gamma>1.2422,(25)$ is satisfied. For $k=\infty$, the inequality is satisfied for $\omega \in(-\infty, \infty)$ and

$$
\lim _{\omega \rightarrow \infty} \frac{\omega^{2}\left((1.8459 \gamma-2.293) \omega^{4}+(2.4733+5.2578 \alpha+0.9298 \gamma) \omega^{2}+0.4924 \alpha\right)}{\left(0.7017 \alpha-0.805 \omega^{2}\right)^{2}+\left(1.325 \omega+2.293 \alpha \omega-\omega^{3}\right)^{2}}=1.8459 \gamma-2.293>0 .
$$

Therefore, the system is absolutely stable for all nonlinearities that belongs to the sector $[\alpha, \infty]$. Note that $\alpha$ can be selected arbitrarily small. This means that the system shown in Fig. 14 with CAPIO is absolutely stable regardless of the rate of the control input. The previous rate limitation required by the circle criterion were conservative and not needed.

\section{Summary}

In this paper, it was presented by simulations that the recently proposed control allocation scheme CAPIO prevents the catastrophic failures due to a PIO event in an inertially cross-coupled aircraft. By helping the aircraft recover from a PIO quickly, CAPIO stabilizes the system. Once the aircraft recovers from the PIO, CAPIO continues to behave like a conventional control allocator. CAPIO achieves this by its dual-behaviour: It minimizes both the errors and the derivatives of the errors between the commanded and achieved accelerations. By minimizing the derivative errors, it prevents effective delay introduction and by minimizing the errors themselves, it helps realizing the commanded accelerations. It was shown that CAPIO works effectively in an inertially cross-coupled MIMO system unlike the previously presented control allocators. The effects of the CAPIO on the closed loop stability was also investigated using a scalar example and it was shown that CAPIO stabilizes a rate saturated system that is nominally, i.e. without a saturation limit, stable.

\section{Acknowledgments}

The authors would like to thank to Dr. Kalmanje S. Krishnakumar of NASA for his suggestion of testing CAPIO for inertially cross-coupled aircraft.

\section{References}

\footnotetext{
${ }^{1}$ Department of Defense Interface Standard, - Flying Qualities of Piloted Aircraft, MIL-STD-1797A, Jan. 1990.

${ }^{2}$ Jeram, G. J. and Prasad, J. V. R., "Tactile Avoidance Cueing For Pilot Induced Oscillation," Proc. AIAA Atmospheric Flight Mechanics Conference and Exhibitt, Austin, TX, Aug. 2003, pp. 1-9.

${ }^{3}$ McRuer, D., "Human Dynamics and Pilot-Induced Oscillations," Minta Martin Lecture, 1992, Massachusetts Institute of Technology, Cambridge, MA.

${ }^{4}$ Yildiz, Y. and Kolmanovsky, I. V., "A Control Allocation Technique to Recover From Pilot-Induced Oscillations (CAPIO) due to Actuator Rate Limiting," Proc. Amer. Control Conf., to be published, 2010.

${ }^{5}$ Klyde, D. H. and Mitchell, D. G., "Investigating The Role of Rate Limiting In Pilot-Induced Oscillations," Proc. AIAA Atmospheric Flight Mechanics Conference and Exhibit, AIAA, Austin, Texas, Aug. 2003, pp. 1-12.

${ }^{6}$ McRuer, D., Klyde, D. H., and Myers, T. T., "Development of a comprehensive PIO theory," Proc. AIAA Atmospheric Flight Mechanics Conference, AIAA, San Diego, CA, July 1996, pp. 581-597.

${ }^{7}$ Klyde, D. H. and Mitchell, D. G., "A PIO Case Study - Lessons Learned Through Analysis," Proc. AIAA Atmospheric Flight Mechanics Conference and Exhibitt, AIAA, San Francisco, CA, Aug. 2005, pp. 1-17.

${ }^{8}$ Deppe, P., Chalk, C., and Shafer, M., "Flight Evaluation of an Aircraft with Side and Centerstick Controllers and Rate-Limited Ailerons," Final rept. 8091-2, Advanced Technology Center, Calspan Corp., Buffalo, NY, April 1994.

${ }^{9}$ Hess, R. A. and Snell, S. A., "Flight Control System Design and Rate Saturating Actuators," Journal of Guidance, Control, and Dynamics, Vol. 20, No. 1, 1997, pp. 90-96.

${ }^{10}$ Snell, S. A. and Hess, R. A., "Robust Decoupled, Flight Control Design with Rate-Saturating Actuators," Journal of Guidance, Control, and Dynamics, Vol. 21, No. 3, 1998, pp. 361-367.

${ }^{11}$ Chapa, M., "A nonlinear Pre-Filter To Prevent Departure and/or Pilot-induced Oscillations (PIO) Due To Actuator Rate Limiting," M.S. thesis AFIT/GAE/ENY/99M-01, Graduate School of Engineering, Air Force Inst. of Technology (AU), Wright-Patterson AFB, OH, March 1999.

${ }^{12}$ Liebst, B. S., Chapa, M. J., and Leggett, D. B., "Nonlinear Prefilter to Prevent pilot-Induced Oscillations due to Actuator Rate Limiting," Journal of Guidance, Control, and Dynamics, Vol. 25, No. 4, 2002, pp. 740-747.

${ }^{13}$ Smith, J. W. and Edwards, J. W., "Design Of A Nonlinear Adaptive Filter For Suppression Of Shuttle Pilot-Induced Oscillation Tendencies," NASA TM-81349, April 1980.

${ }^{14}$ Koper, J., "An Approach for Compensating Actuator Rate Saturation," Interim rept. nadc-87120-60, Air Vehicle and Crew systems Technology Dept., Naval Air Development Center, Warminster, PA, Aug. 1987.
} 
${ }^{15}$ Rundqwist, L. and Hillgren, R., "Phase Compensation of Rate Limiters in JAS 39 Gripen," Proc. AIAA Atmospheric Flight Mechanics Conference, AIAA, San Diego, CA, July 1996, pp. 69-79.

${ }^{16}$ Hanke, D., "Phase Compensation: A means of Preventing Aircraft-Pilot Coupling Caused by Rate Limitation," Tech. rep., DLR - Forschungsbericht 98-15, 1998.

${ }^{17}$ Hanke, D., "Handling Qualities Analysis on Rate Limiting elements in Flight Control systems," Proc. Flight vehicle Integration Panel workshop in Pilot Induced Oscillations, AGARD-AR-335, Feb. 1995.

${ }^{18}$ Bodson, M., "Evaluation of Optimization Methods for Control Allocation," Proc. AIAA Guidance, Navigation, and Control Conference and Exhibit, AIAA, Montreal, CA, Aug. 2001, pp. 1-15.

${ }^{19}$ Durham, W. C. and Bordignon, K. A., "Multiple Control Effector Rate Limiting," Journal of Guidance, Control, and Dynamics, Vol. 19, No. 1, 1996, pp. 3037.

${ }^{20}$ Blakelock, J. H., Automatic Control of Aircraft and Missiles, John Wiley \& Sons, 2nd ed., 1991.

${ }^{21}$ Nguyen, N., Krishnakumar, K., Kaneshige, J., and Nespeca, P., "Dynamics and Adaptive Control for Stability Recovery of Damaged Asymmetric Aircraft," Proc. AIAA Guidance. Navigation, and Control Conference, Keystone, CO, 2006, pp. 1-24.

${ }^{22}$ Ola Harkegard, S. and Glad, T., "Resolving Actuator Redundancy - optimal control vs. control allocation," Automatica, Vol. 41, 2005, pp. 137-144.

${ }^{23}$ Aerodata Model in Research Environment (ADMIRE), version 3.4h., Swedish Defence Research Agency (FOI), URL: www.foi.se/admire, 2003.

${ }^{24}$ Backstrm, H., "Report on the usage of the Generic Aerodata Model," Technical report, Saab Aircraft AB, May 1997.

${ }^{25}$ Khalil, K. H., Nonlinear Systems, Prentice-Hall, Upper Saddle River, NJ, 3rd ed., 2002. 